\title{
SMALL FOR GESTATIONAL AGE
}

\author{
Geoffrey Chamberlain
}
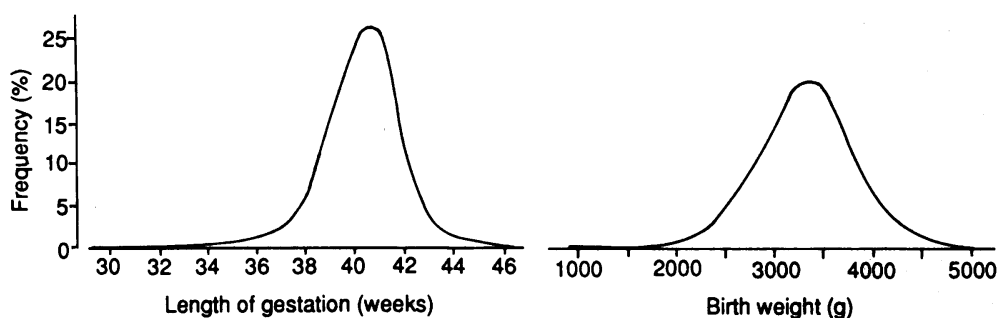

Distribution of length of gestation and birth weight (singletons, last menstrual period certain).

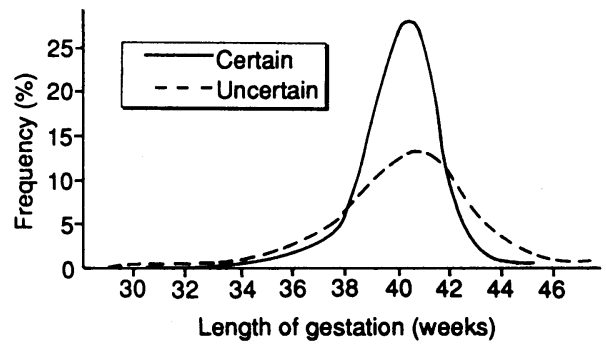

Distribution of length of gestation by knowledge of last menstrual period (singletons).

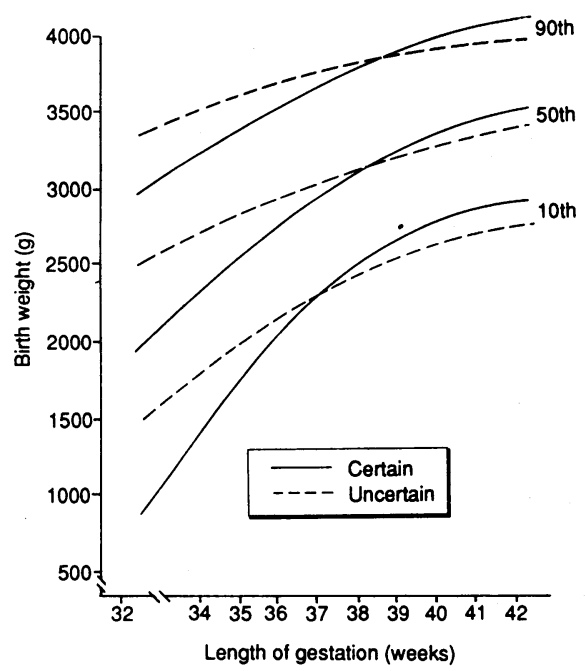

Centiles of birth weight by length of gestation and certainty of last menstrual period (singletons).

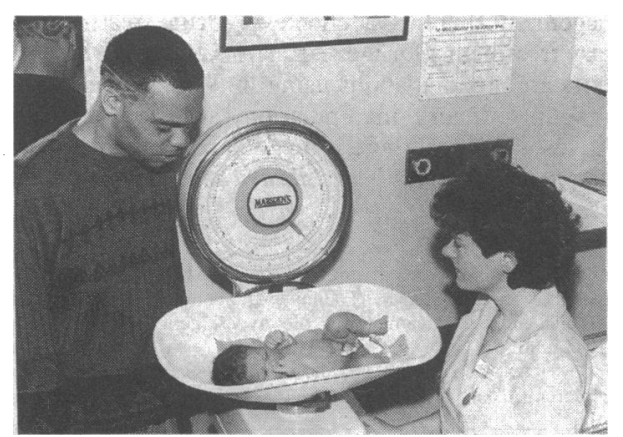

The diagnosis of a small fetus is made more specific by examining the ratio of birth weight (or estimated birth weight) to gestational age. Both these measures have inherent problems.

Obstetricians estimate fetal weight either clinically or from measuring ultrasound determined diameters of the fetus in utero. Gestational age is derived from the mother's menstrual dates, which are usually confirmed by an ultrasound scan measuring the biparietal diameter performed before 20 weeks. In the British Births Survey (1970) of 16797 women only 13634 $(81 \cdot 1 \%)$ were sure of their dates. The figures show the distribution of length of gestation for women according to whether they were sure of their dates and that the frequency of heavier babies was increased among those uncertain of the date of their last menstrual period. All women with unsure dates should have gestational age established by ultrasonography, as should those in whom there is a discrepancy between the dates derived from the last menstrual period and fetal size in early pregnancy. Obstetricians consider a baby to be small for gestational age when abdominal circumference readings fall below the second standard deviation of the mean; this is approximately $2 \cdot 3$ centile on serial ultrasonography.

Paediatricians can weigh the baby and have a more precise measure, although even this varies slightly with the conditions of weighing and when it is done. Gestational age is obtained from the obstetrician by one of the previously mentioned measures or from Dubowitz scoring. The data are plotted on a specific centile chart; various groups of paediatricians take small for gestational age as being below the 10th, the fifth, or the third centile. It is very important when examining data to know which of these measures was used. The 10th centile is rather crude and will include many babies whose growth has not actually been affected by placental bed disease. 


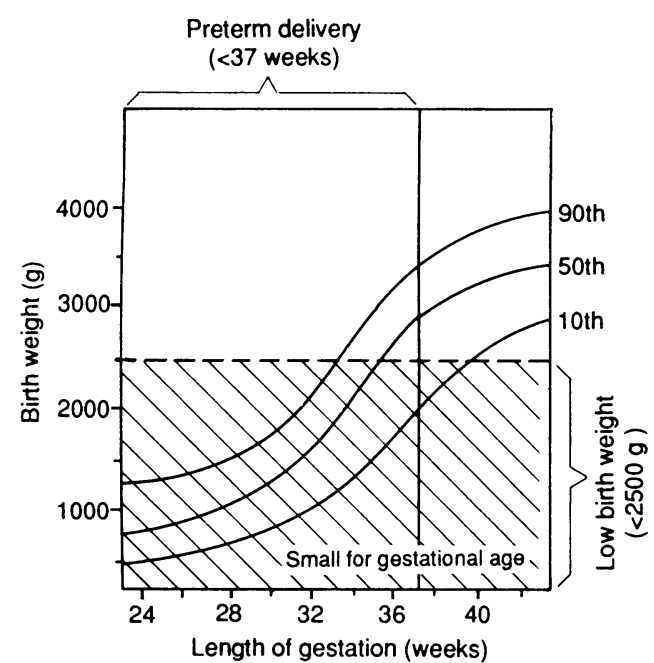

The relation between preterm and low birth weight babies. Babies who are small for gestational age fall under the 10th centile.
Much simpler was the old fashioned measure of prematurity taking a cut off point of a birth weight of less than $2500 \mathrm{~g}$. Unfortunately, this includes small babies whose birth weight is appropriate for their gestational age and those who are small for their gestational age, two very different groups in clinical medicine. For example, babies born with a birth weight below $2500 \mathrm{~g}$ make up about $7 \%$ of the newborn population in the United Kingdom, about 3\% in Sweden, and almost $11 \%$ in Hungary. Such mixed data would make a nonsense of studying the influences on fetal growth and so the definition of small for gestational age relating birth weight to length of intrauterine stay stands for the moment.

\section{Causes}

In the United Kingdom most of the energy required by a pregnant woman can come from an ordinary diet, with little need for supplementation

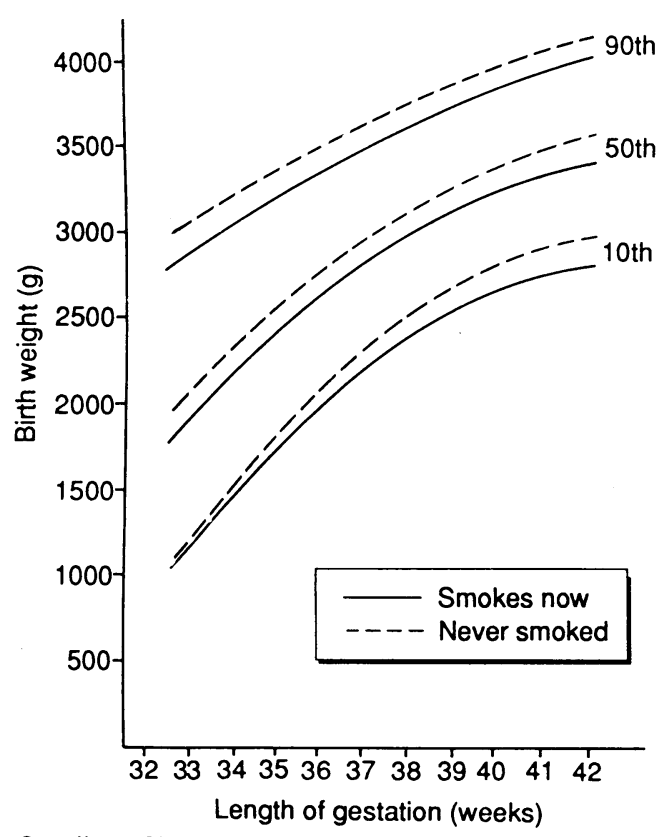

Centiles of birth weight by length of gestation and mother's smoking habit (singletons, last menstrual period certain).

\section{Genetic abnormalities}

Genetic abnormalities are an identifiable but not very common factor causing growth retardation. Trisomy 21 is the commonest example, though osteogenesis imperfecta, Potter's syndrome, and anencephaly may all be associated with intrauterine growth retardation. Other congenital malformations not yet proved to have a chromosome component are commonly found in fetuses that are small for gestational age; among them are gastrointestinal abnormalities such as atresia of the duodenum, gastroschisis, and omphalocele.

\section{Maternal nutrition}

In the United Kingdom the effect of maternal nutrition on low birth weight is probably small. Extremes of starvation associated with small babies are rare in Britain. During a pregnancy about 80000 kilocalories $(335 \mathrm{MJ})$ of extra energy is required, of which 36000 kilocalories $(150 \mathrm{MJ})$ is for maintenance metabolism. Much of this can come from the everyday diet, and among well nourished women requirements change little for the first 10 weeks of pregnancy. Thence requirements gradually increase, but ordinary variations in food intake are unlikely to affect events. It is unwise to recommend that a mother eat for two in order to produce a larger baby as there are many covariables in nutrition other than what she eats.

\section{Intrauterine infection}

Intrauterine infection may be viral or bacterial. Some $60 \%$ of babies with congenital rubella are born below the 10th centile of weight for gestation. Cytomegalovirus and toxoplasmosis (much less common in this country than in Europe) are associated with growth retardation in about $40 \%$ of affected infants. Malaria, ubiquitous in many tropical countries, causes a massive accumulation of monocytes in the intravillus space, which can lead to a fetus being small for gestational age. Syphilis, a rare disease in this country, used to be associated with babies being small for gestational age but the few babies now born with congenital syphilis do not exhibit this notably.

\section{Drugs}

Drugs may be a cause of babies being small for gestational age. The commonest cases in the United Kingdom are the results of burning tobacco during cigarette smoking. Small for gestational age babies and association with smoking is well documented; the number of affected babies whose growth drops below the 10th centile increases during the last weeks of gestation. The effect of alcohol is difficult to sort out. At the extreme end of 


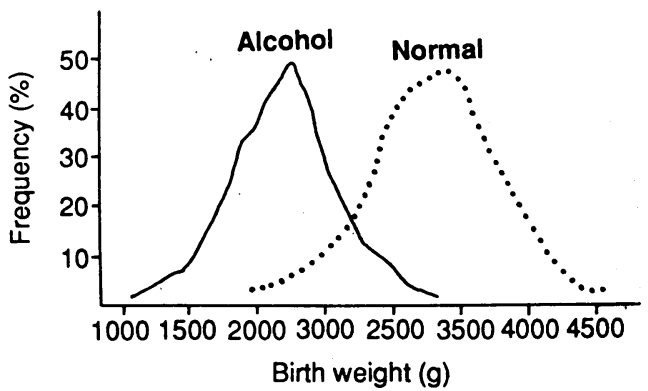

Distribution of birth weight in a normal population of women and in one consisting of women who drank more than $\mathbf{4 5}$ units of alcohol a week (heavy drinking).

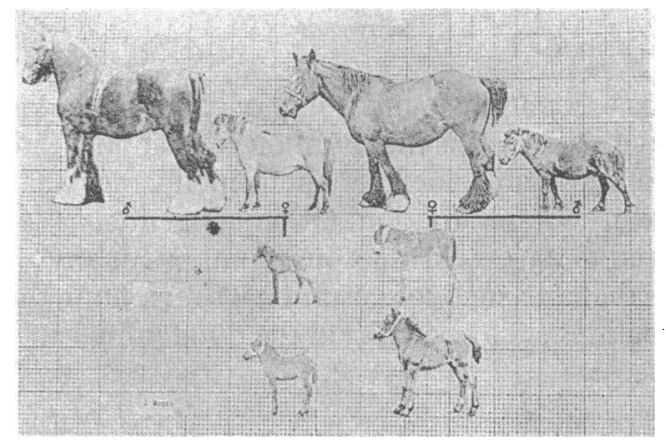

The effects of sire and mare on the size of offspring is shown in this 1938 experiment in which Shire horses and Shetland ponies were mated. The maternal influence predominates. the range (women drinking more than 45 units of alcohol a week) many babies are born with the fetal alcohol syndrome and a distinctly reduced birth weight. At lower intakes of alcohol other covariables come into play. A deficient maternal diet and increased cigarette smoking are two well known ones. In some studies multivariant analyses show that the main causal factor associated with low birth weight is not alcohol intake but cigarette smoking. The whole lifestyle is probably the important factor in this case. Some doctors consider that smoking in pregnancy is the most important single cause of low birth weight, which is the most important cause of death and illness in the first weeks of life.

The intake of narcotic drugs is commonly associated with low birth weight but, again, the total lifestyle of the woman may be the real factor. For this reason, perhaps, an increased incidence of small for gestational age babies persists with methadone users.

Therapeutic drugs such as carbamazepine and the valproates have been associated with an increased incidence of small for gestational age babies, as are the more powerful antiviral drugs such as azathioprine. Therapeutically powerful drugs are not given in pregnancy unless to treat a serious maternal medical condition, which in itself may affect nutrition or metabolism of the mother and growth of the fetus.

\section{Hypertension}

Probably one of the major current causes of babies being small for gestational age in the United Kingdom is hypertension in the mother, either pregnancy induced or pre-existing. After other features have been taken into account such types of hypertension are responsible for about a third of all cases of intrauterine growth retardation. The effects of hypertension are made worse when raised blood pressure is associated with proteinuria, implying a greater reduction of the maternal perfusion of the placental bed. The duration of the condition also has an effect - for example, $80 \%$ of mothers who have proteinuric pregnancy induced hypertension before the 34 th week of pregnancy have infants with a birth weight below the 10 th centile.

\section{Other factors}

The maternal body habitus is not a critical factor in babies being small for gestational age, but big women do produce larger children. The father's influence is less important, classically shown in the 1938 study of Walton and Hammond on Shire horses and Shetland ponies. The altitude at which a woman lives in pregnancy has an effect on fetal growth, particularly if she is not used to high altitudes.

\section{Diagnosis}

- Symmetrical growth retardation occurs in low birthweight babies when the ratio of head to abdominal circumference is normal

- Asymmetrical growth retardation occurs in low birthweight babies when the ratio of head to abdominal circumference is increased
Extreme examples of fetuses that are severely small for gestational age can sometimes be diagnosed by palpation. This is most likely if the same observer sees the woman at each antenatal visit and uses the written records of previous visits longitudinally. In several control studies false positive rates as high as $\mathbf{5 0 \%}$ and low predictive values have been found in the clinical estimation of intrauterine growth retardation.

Sometimes the lack of amniotic fluid is diagnosed more readily; oligohydramnios accompanies fetuses that are small for gestational age and therefore may lead observers to investigate more swiftly than when fetal size has been estimated clinically.

Most fetuses that are small for gestational age are diagnosed in this country by ultrasonography. When a good estimate of gestational age in early pregnancy has been obtained and fetal abnormalities have been excluded, ultrasound scans can give valid measures of fetal growth. Scans of the body area or circumference at the level of the umbilical vessels give a measure of liver growth. Another measure of somatic growth is femur length. 


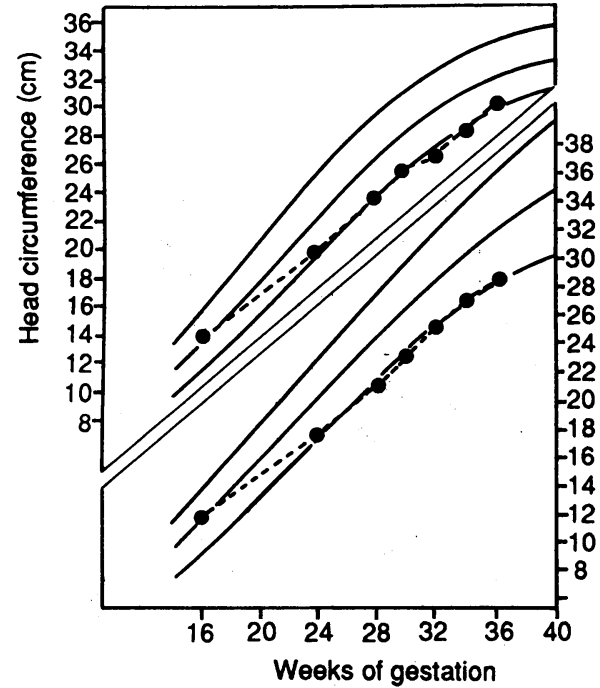

Ultrasound measures of the head and abdominal circumference. Although growth rates are diminished, they fall at the same ratesymmetrical growth retardation.

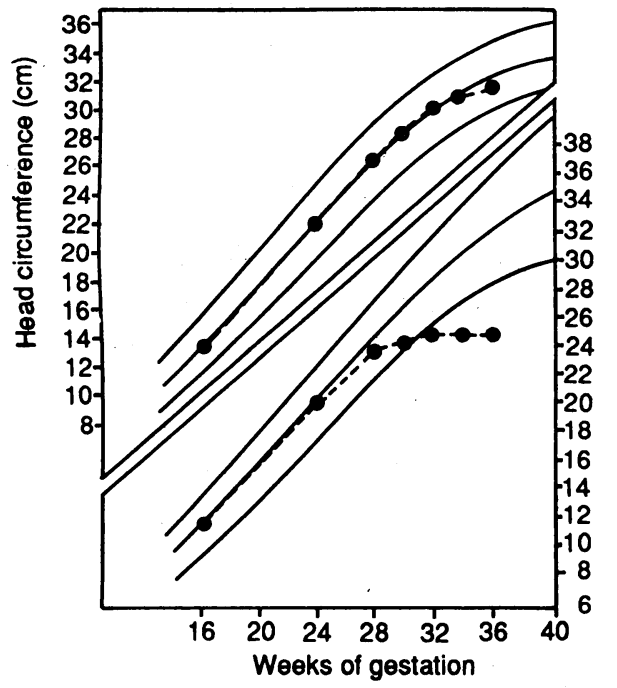

Ultrasound measures of head and abdominal circumference. Abdominal growth slows more than head growth-asymmetrical growth retardation.

\section{Treatment}

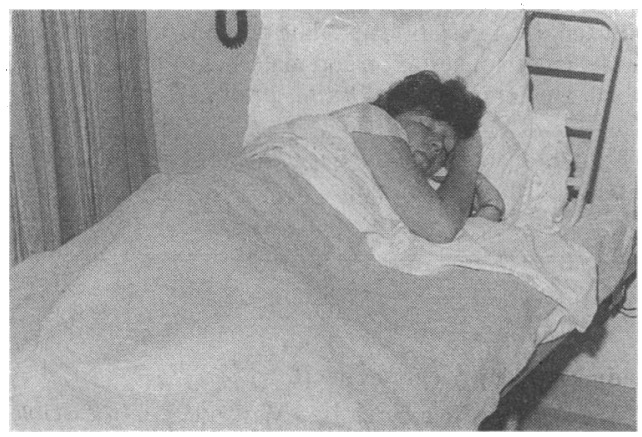

Woman lying in the left lateral position.
Fetuses with small abdominal circumferences should have their head circumference measured and the ratio of head to abdominal circumference derived. A small for gestational age fetus with a normal ratio of head to abdominal circumference tends to be a perfect miniature (Bonsai baby) and is normal, representing the lower end of biological variation. Such fetuses, however, may also be associated with chromosomal anomalies, drugs, infection, or malnutrition. Fetuses suffering from placental insufficiency tend to preserve growth of the head at the expense of the body because of the protective mechanism shunting blood to the brain. Such babies suffer hypoxic and ischaemic brain damage in pregnancy before labour, but it may be only in labour that the fetal heart rate shows this.

Estimating fetal weight in utero uses one or more ultrasound measurements. All methods have a minimum error of $150 \mathrm{~g} / \mathrm{kg}$ in relation to true fetal weight and are therefore better than clinical ways of predicting smaller babies.

Babies who are symmetrically small for gestational age (normal ratio of head to abdominal circumference) usually show normal growth subsequently and therefore only require serial ultrasound examination. Deciding which fetuses to examine karyometrically or for infection is difficult, but often an abnormal fetus will show minor structural markers such as cardiac defects, occipital fat pads, abnormal hand positions, or intracranial calcification. Asymmetrical small for gestational age fetuses used to be monitored by cardiotocography. Doppler studies of the uterine and umbilical circulation are rapidly replacing these (see article on checking for fetal wellbeing-II).

There is little help from assessing oestrogen concentrations in small for gestational age fetuses, although many obstetric departments have forgotten to stop using such tests. In an individual fetus longitudinal increases in plasma oestriol concentration are a reassuring sign, but the converse is not crisp enough to be a guide for action and positive predictive values are low (see article on checking for fetal wellbeing-II).

Small for gestational age fetuses may be screened by using early ultrasonography to confirm gestational age and later to confirm growth. Finer tuning is possible by Doppler measurement of the afferent supply to the placental bed, with later changes in flow along the umbilical vessels giving a more precise warning of fetal state. In practice these are covered by an ultrasound reading of the biparietal diameter at 16-20 weeks to confirm gestation and a second scan of abdominal circumference at 32-36 weeks to check growth.

Mothers whose fetuses are at greater risk of intrauterine growth retardation often have several ultrasound readings performed in later pregnancy. Such women include those with a history of perinatal death and of intrauterine growth retardation as well as those in whom the fetus is exposed to some of the aetiological factors considered earlier and where oligohydramnios may give a clue.

The ultimate treatment of a fetus with impaired growth associated with the placental bed is delivery. Diagnosis encapsulates the fact that the baby is getting insufficient nutrition to grow at the expected rate so that in labour it will be in greater danger of oxygen deprivation. Removal from the hostile environment is the ultimate answer, but this might not be wise in early gestation (24-30 weeks); efforts are made to improve the blood supply to the placental bed.

Early work with abdominal decompression to improve uterine blood supply was unrewarding. Biopharmacological methods of increasing the diameter of the afferent vessels of the placental bed with $\beta$ mimetics have been tried but have generally proved to be unsuccessful. The use of aspirin is currently under investigation in a multicentre trial (CLASP), and some general practitioners' patients may be a part of the study. Bed rest, particularly with the woman lying on her left side for some hours a day, should theoretically improve placental perfusion, but Doppler studies show little evidence for its effectiveness. Measures to restore the plasma volume and to give adequate hydration seem useful theoretically as they should decrease viscosity and lead to an improvement of intrauterine blood flow. Again, theory is not matched by practice. 


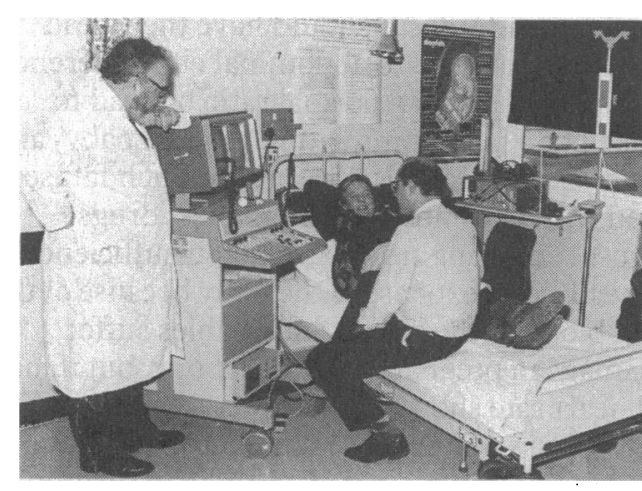

Corner of a fetal measurement laboratory.

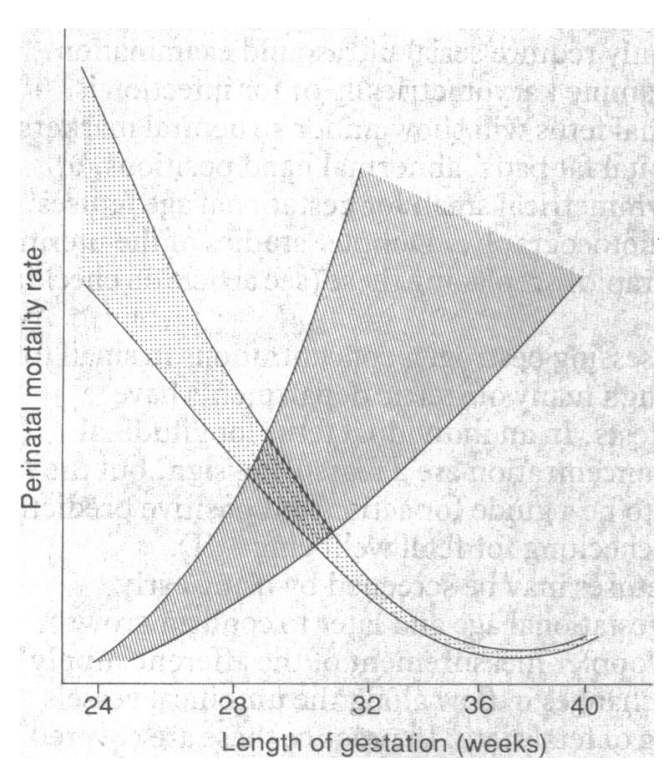

The relative risks to a fetus of staying in the uterus on the wrong side of a poor placental bed perfusion system risks of being delivered too soon
In fetuses that are symmetrically small for gestational age correction and reversal of some of the causal factors might have helped, but it is too late to do this when the fetus is detectably small for gestational age. For example, curtailment of cigarette smoking should happen in early pregnancy. Such curtailment in the first 16 weeks allows fetuses to follow a normal growth pattern rather than that of growth retarded babies of smoking mothers.

The mother of a fetus that is small for gestational age should attend a hospital that has a capacity for more precise diagnosis and where special ultrasound and Doppler measurements are available. Many tertiary referral centres have a fetal measurement laboratory run on a day care basis. Women who live near large hospitals with such centres can still be outpatients while having full surveillance. If they live away from the centre, however, they may have to be transferred and become inpatients; this is the keystone of the in utero transfer system widespread in the United Kingdom. Probably a third of the women admitted as in utero transfers have fetuses that are small for gestational age as their indication for admission.

The double ultrasonographic surveillance of fetal growth and placental bed blood flow allows a more precise assessment to be made of fetal progress. Prospective frequent and regular consultations with the neonatal paediatrician who will be involved is essential. The fetus must be delivered at the most appropriate time by the most appropriate method. The time depends on weighing up the risks of keeping the fetus inside the uterusthat is, those of diminished placental bed perfusion-against the risks of being outside - that is, the risks of immaturity and survival in a good intensive care neonatal unit. The critical gestational age for these decisions is being pushed back all the time; now the worrying time for most obstetricians and neonatal paediatricians is 24-28 weeks. Once a pregnancy passes 30 weeks the concern is much less, although the respiratory distress syndrome can still cause illness after delivery.

\section{Conclusions}

The diagnosis, causes, and management of small for gestational age fetuses are all still uncertain. The best management is prevention
It must be remembered that the definitions of small for gestational age are used imprecisely and much that was thought to be known about its causation depended on data that were not mutually comparable. Until Doppler measurement the measures of fetal wellbeing were also inexact; even Doppler ultrasonography is not the last word on the subject. The ultimate management depends on avoiding trouble. Maybe we are overprotective of fetuses that are small for gestational age, but it is the best that we can do in 1991.

The figures showing the distribution of birth weight, the distribution of the length of gestation, the centiles of birth weight by length of gestation and date of the last menstrual period, and the centiles of birth weight by length of gestation and maternal smoking habit are reproduced by permission of Butterworth Heinemann from British Births 1970 by R Chamberlain and G Chamberlain.

Professor Geoffrey Chamberlain, FRCOG, is chairman of the department of obstetrics and gynaecology at St George's Hospital Medical School, London.

\section{Correction}

ABC of Antenatal Care: Vaginal bleeding in early pregnancy -II

An error occurred in this article by Professor Geoffrey Chamberlain (18 May, p 1195). In the table of the symptoms and signs of ectopic pregnancy the symptoms of unruptured and ruptured ectopic pregnancy are transposed. The table should read that the symptoms of an unruptured ectopic pregnancy have a gradual onset and consist of a dull ache over days and that those of a ruptured ectopic pregnancy have a sudden onset and consist of severe pain over minutes (and not vice versa as published). 